\title{
A Multistandard Frequency Offset Synchronization Scheme for 802.11n, 802.16d, LTE, and DVB-T/H Systems
}

\author{
Javier González-Bayón, ${ }^{1}$ Carlos Carreras, ${ }^{1}$ and Ove Edfors ${ }^{2}$ \\ ${ }^{1}$ Departamento de Ingeniería Electrónica, E.T.S.I. Telecomunicación, Universidad Politécnica Madrid, 28040 Madrid, Spain \\ ${ }^{2}$ Department of Electrical and Information Technology, Lund University, 22100 Lund, Sweden \\ Correspondence should be addressed to Javier González-Bayón, javier@die.upm.es
}

Received 21 October 2009; Accepted 24 December 2009

Academic Editor: Francisco Falcone

Copyright (C) 2010 Javier González-Bayón et al. This is an open access article distributed under the Creative Commons Attribution License, which permits unrestricted use, distribution, and reproduction in any medium, provided the original work is properly cited.

\begin{abstract}
Carrier frequency offset (CFO) synchronization is a crucial issue in the implementation of orthogonal frequency division multiplexing (OFDM) systems. Since current technology tends to implement different standards in the same wireless device, a common frequency synchronization structure is desirable. Knowledge of the physical frame and performance and cost system requirements are needed to choose the most suitable scheme. This paper analyzes the performance and FPGA resource requirements of several data-aided (DA) and decision-directed (DD) schemes for four wireless standards: 802.11n, 802.16d, LTE, and DVB-T/H. Performance results of the different methods are shown as BER plots and their resource requirements are evaluated in terms of the number of computations and operators that are needed for each scheme. As a result, a common architecture for the four standards is proposed. It improves the overall performance of the best of the schemes when the four standards are considered while reducing the required resources by $50 \%$.
\end{abstract}

\section{Introduction}

OFDM has been the focus of a wide variety of studies in wireless communication systems because of its high transmission capability and its robustness to the effects of frequency-selective multipath channels. Several existing and upcoming standards, among them are WiFi 802.11n [1], WiMAX 802.16d [2], LTE [3], and DVB-T/H [4, 5], are based on the OFDM concept. It is expected that several of them will coexist and, in many cases, operate concurrently on the same wireless terminal. This opens up for receiver/transmitter algorithm design where the basic algorithm structure is shared between the different OFDMbased standards, allowing for both efficient implementations and efficient use of resources on a common baseband processing platform. Several approaches to multistandard solutions can be found in the literature [6-9], but none of them deals with the synchronization problem in detail.

It is well known that OFDM systems are more sensitive to an offset in the carrier frequency than single carrier schemes at the same bit rate. This CFO causes loss of orthogonality of the multiplexed signals creating intercarrier interference (ICI) and introducing a constant increment in the phase of the samples.

Frequency synchronization is often performed in two phases: acquisition and tracking. At the start of the sequence the acquisition stage is used to perform a first estimation of the CFO of the signal [10-14]. In a circuit-switched system the acquisition phase can be fairly long since it only represents a small percentage of the total transmitted sequence. Some systems like LTE, DVB, and cellular systems are circuitswitched. In packet-switched systems, as $802.16 \mathrm{~d}$ and $802.11 \mathrm{n}$, the acquisition phase is more important since the transmission sequences are short. The most common approach in such systems is to use a preamble for acquisition. As it will be shown, the acquisition stage is a well-defined task that can be easily adapted to all standards being considered. Therefore, the paper focuses specially on the tracking stage.

After acquisition, the problem of tracking has to be solved. Since acquisition is never performed perfectly and conditions are not static in a real system, there still remains a residual CFO that needs to be corrected. The tracking stage 
can be non-data-aided [15], when no extra information is included in the transmitted data (as in DD methods) or data aided $[12,16]$, when periodically transmitted training symbols and/or known pilot subcarriers are used.

In this paper, different frequency synchronization schemes are evaluated for the addressed standards with an explicit aim to reuse as much as possible the algorithm structure when switching between standards because of the limited resources available in the target architecture. Therefore, algorithm and architectural design are approached together from the beginning of the design flow. In this study, FPGAs have been selected as target architecture for these systems because of their support for reconfigurability, parallelism, and increased performance over software-based (e.g., DSP) solutions.

The main contributions of this paper are as following:

(1) detailed performance analysis of CFO synchronization schemes (mainstream and alternative) for four current wireless communications standards,

(2) comparative evaluation of their computational requirements,

(3) proposal of feasible architectures for multistandard devices.

The paper is structured as follows. The OFDM signal and the different standard frames are introduced in Sections 2 and 3. The acquisition and the different tracking schemes are presented in Sections 4 and 5. BER results for the different standards are given in Section 6. Implementation issues are considered in Section 7. Finally, Section 8 concludes the paper.

\section{The OFDM Signal}

The baseband scheme of a digitally implemented OFDM transmission system with CFO correction enabled is provided in Figure 1. Considering an OFDM system, the data source emits symbols $\left(d_{i}\right)$ which belong to a BPSK, QPSK, 16-QAM, or 64-QAM constellation and are assumed to be equiprobable and statistically independent. The sequence $d_{i}$ is serial to parallel converted into blocks of $N$ symbols $\left(d_{k, l}\right.$ denotes the $k_{\text {th }}$ symbol of $l_{\text {th }}$ block where $k=0, \ldots, N-1$, and $l=-\infty, \ldots,+\infty)$. These blocks are generated with period $T_{s}=T+T_{g}$ ( $T$ : useful period, $T_{g}$ : guard interval). After the inverse FFT (IFFT) is applied to each block with period $T_{s}$, a cyclic prefix (CP) is inserted by prefixing the resulting $N$ samples $\left(s_{n, l}^{\prime}, k=0, \ldots, N-1\right)$ with a replica of the last $N_{g}$ samples. Thus, each block is made of $N_{s}=N+N_{g}$ samples called an "OFDM symbol".

Since the carrier frequency difference between the transmitter and the receiver $\Delta f$ can be modeled as a timevariant phase offset, $e^{j 2 \pi \Delta f t}$, the received OFDM signal can be represented as

$$
r(t)=e^{j 2 \pi \Delta f t} s(t) * h(t, \tau)+w(t)
$$

where $w(t)$ is the additive white Gaussian noise (AWGN), $s(t)$ is the transmitted baseband OFDM signal, $h(t, \tau)$ is the channel impulse response with $\tau$ being the delay spread, and “*” denotes linear convolution.
Assuming that $r(t)$ is sampled at the transmit interval $T$ with perfect timing, the samples blocked for the $l$ th FFT are

$$
r_{n, l}=r\left[\left(n+N_{g}+l N_{s}\right) T\right], \quad 0 \leq k<N,-\infty<l<+\infty .
$$

The resulting samples from the FFT obtained in (2) are [17]

$$
\begin{aligned}
c_{k, l}= & e^{j \pi((N-1) / N) \varepsilon} e^{j 2 \pi\left(\left(l N_{s}+N_{g}\right) / N\right) \varepsilon} \frac{\sin (\pi \varepsilon)}{N \sin (\pi \varepsilon / N)} H_{k, l} d_{k, l} \\
& +\mathrm{ICI}_{k, l}+W_{k, l}, \quad 0 \leq k<N,-\infty<l<+\infty
\end{aligned}
$$

where $\varepsilon=\Delta f T$ is the CFO normalized with respect to the subcarrier spacing. Likewise, $H_{k, l}$ is the channel coefficient on the $k_{\text {th }}$ subcarrier with the assumption that the channel is stationary during at least one symbol, $\mathrm{ICI}_{k, l}$ is the intercarrier interference noise due to loss of orthogonality and, $W_{k, l}$ is a zero-mean stationary complex process. The first term is the data value $d_{k, l}$ modified by the channel transfer function, experiencing an amplitude reduction and phase shift due to the frequency offset.

\section{The Standard Frames}

The IEEE $802.11 \mathrm{n}$ standard is the latest in the 802.11 family. It adds extra functionality and provides better spectral efficiency. High data rates are achieved through space division multiplexing and multiple-input-multiple-output (MIMO) antenna configurations, though this paper will focus on the single input and output (SISO) antenna case. This standard defines a physical layer that can use 64 or 128 subcarriers with local oscillator frequencies of $2.4 \mathrm{GHz}$ or $5 \mathrm{GHz}$. Also, it can operate in three modes: legacy, high throughput, and mixed. This paper focuses on the mixed and legacy modes where the preamble is composed of repeated patterns in the time domain called short training field (STF) and long training field (LTF) and other signal field preambles, as illustrated in Figure 2. The correlation properties of STF and LTF allow CFO estimation in the acquisition stage. Also, 802.11n allocates a number of boosted pilot subcarriers ( 4 or 6 ) in the data symbols for channel estimation and synchronization purposes.

The IEEE 802.16d standard (also known as fixed WiMAX) defines a physical layer that uses 256 subcarriers which are modulated with BPSK, QPSK, 16-QAM, or 64QAM constellations. The transmission according to IEEE 802.16 is done in bursts, similarly to 802.11 n. The WiMAX OFDM preamble is defined differently for uplink and downlink communications [2]. In both cases, the time domain signal of the preamble has a repeated pattern. The long preamble, used for downlink, consists of two symbols: a $4 \times 64$ pattern symbol, where a 64 -sample pattern is repeated 4 times, and a $2 \times 128$ pattern symbol with two repetitions of a 128-sample pattern. The uplink uses a short preamble with just a $2 \times 128$ pattern symbol. This work will focus on the uplink frame. Eight boosted subcarriers are allocated for pilot signals and a number of the highest and lowest 


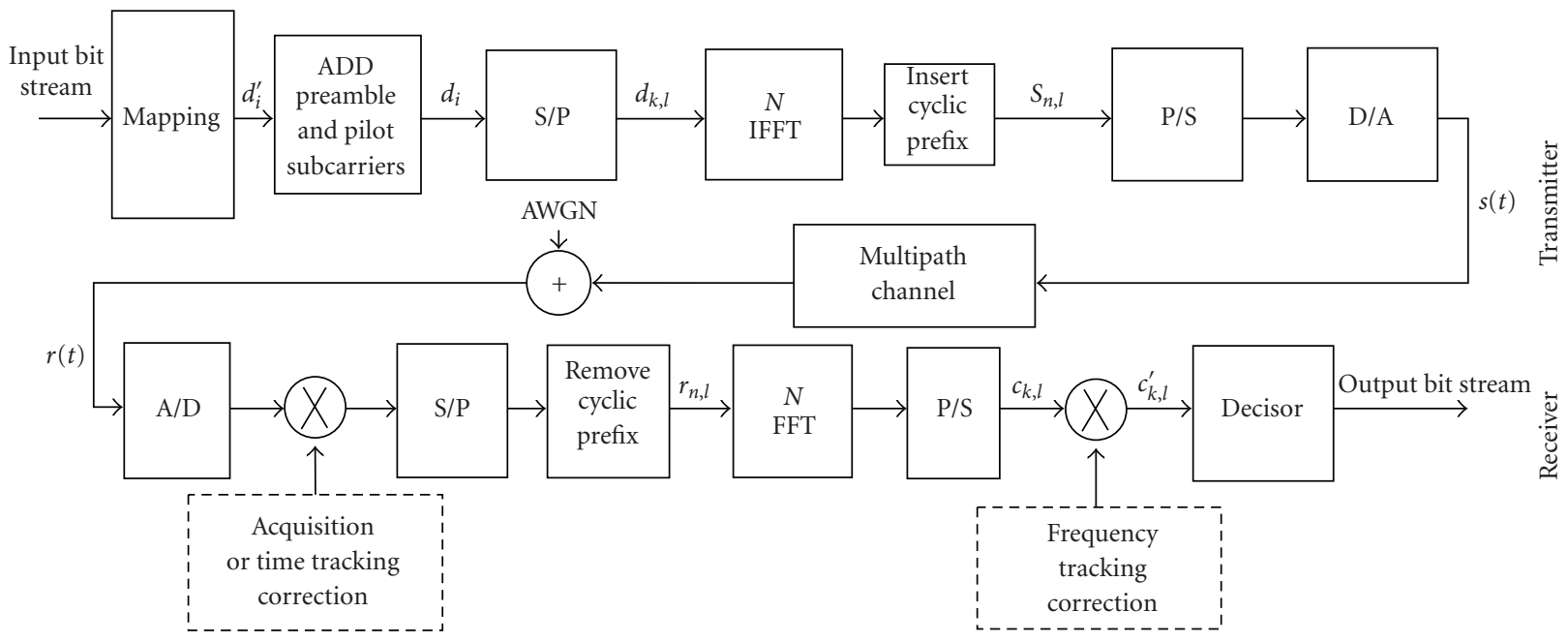

Figure 1: OFDM block diagram.

\begin{tabular}{|c|c|c|c|c|c|c|}
\hline L-STF & L-LTF & L-SIG & HT-SIG & HT-STF & HT-LTFs & Data \\
\hline
\end{tabular}

Figure 2: Preamble for 802.11 n mixed mode.

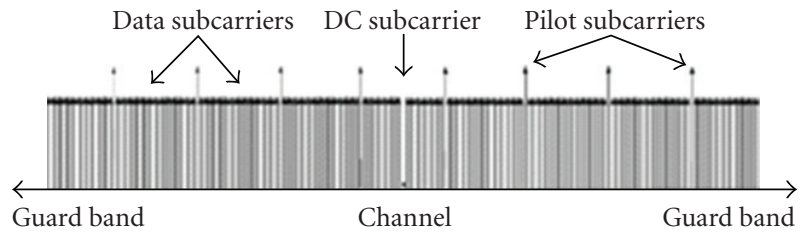

FIgURE 3: Frequency domain for 802.16d.

frequency subcarriers are null. The shape of the WiMAX OFDM signal in the frequency domain is shown in Figure 3.

LTE is a project belonging to the Third Generation Partnership Project (3GPP) to improve the Universal Mobile Telecommunications Systems (UMTS) and to cope with future communications requirements. LTE uses OFDM in the downlink which results in high spectral efficiency. It is also designed to be flexible in the channel allocation. In contrast to packet-oriented networks, LTE does not include a preamble to facilitate timing and frequency synchronization. Instead, pilot subcarriers are embedded in the frame as shown in Figure 4. In the normal mode, pilot subcarriers are transmitted every six subcarriers during the first and fifth OFDM symbols of each slot. This paper deals exclusively with the Frequency Division Duplex (FDD) mode defined in the standard.

Systems using DVB standards focus on digital television and data services. Even though the DVB-T standard is prepared for mobile reception, there are some factors that have to be considered when the end device is running under limited power constraints. This was the major motivation to develop a new broadcast standard aimed for handheld devices. This standard is denoted as DVB-H. It contains two major additions to the DVB-T standard, namely, time slicing and a new mode of operation called $4 \mathrm{~K}$. However, the physical frame has the same structure as in DVB-T. Therefore, similar synchronization schemes can be performed for both standards. DVB-H specifies three possible OFDM modes $(2 \mathrm{~K}, 4 \mathrm{~K}$, and $8 \mathrm{~K})$. As with LTE, $\mathrm{DVB}-\mathrm{T} / \mathrm{H}$ does not include a preamble for timing and frequency synchronization purposes. It defines dedicated synchronization subcarriers embedded into the OFDM data stream: continual (periodicity in the time domain) and scattered pilot subcarriers (periodicity in the frequency domain). Both continual and scattered pilots are transmitted at a boosted power level and their position can be observed in Figure 5.

In order to choose a suitable frequency synchronization scheme, special attention must be paid to the reference OFDM symbols and pilot subcarriers. In $802.11 \mathrm{n}$ and $802.16 \mathrm{~d}$ there is a preamble amended at the beginning of the frame, whereas in LTE and DVB-T/H there is no preamble. Therefore, correlation properties introduced by the $\mathrm{CP}$ should be used in the acquisition stage for these two standards. Continual pilot subcarriers are defined in $802.11 \mathrm{n}, 802.16 \mathrm{~d}$, and DVB-T/H but LTE only includes pilot subcarriers at some specific OFDM symbols. Thus, data-aided tracking performance would perform better in $802.11 \mathrm{n}, 802.16 \mathrm{~d}$, and DVB-T/H than in LTE if the pilots are used for tracking purposes. From these observations, it seems that using a decision-directed algorithm in the 


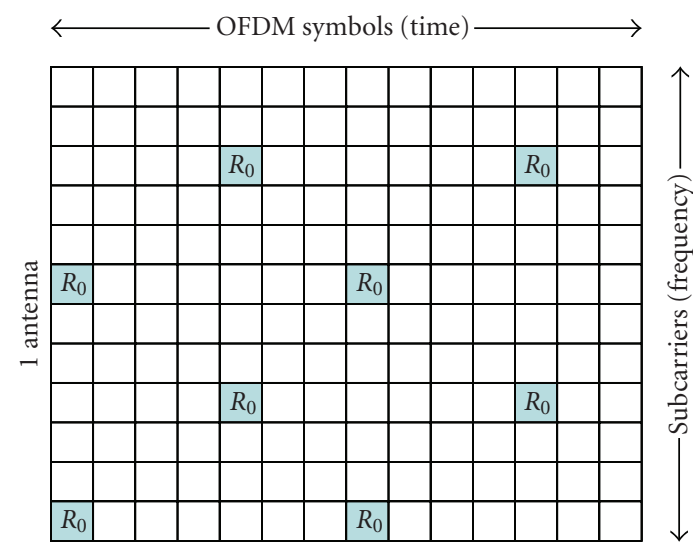

FIgURE 4: Reference pilots in LTE.

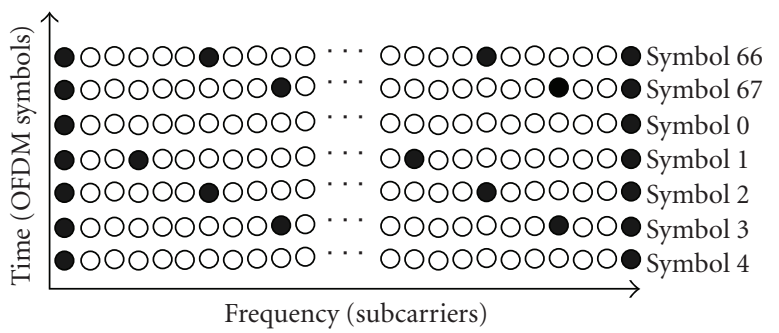

- Boosted pilot

O Data

Figure 5: DVB-T/H pilot structure.

tracking stage would lead to a more homogeneous approach in a multistandard system.

\section{CFO Acquisition Schemes}

Most of the solutions for acquisition use the aid of pilot symbols, which are assumed to be known at the receiver. An alternative technique is to use the redundant information included in the CP [10]. Furthermore, CFO acquisition can be divided in two steps as explained in $[11,12]$. In the first step, the fractional part of the CFO is estimated and corrected, allowing for the integer part of the CFO to be estimated and corrected in the second step.

The $802.11 \mathrm{n}$ and $802.16 \mathrm{~d}$ standards include a preamble at the beginning of the frame. This preamble has an OFDM symbol with a repeated pattern in the time domain. The Moose algorithm [13] can be used to perform the fractional acquisition stage by using this symbol. Let there be $L$ complex samples in each half of the training symbol, and let the correlation parts be

$$
P=\sum_{m=0}^{L-1}\left(r_{m}^{*} r_{m+L}\right) .
$$

Considering the LTF symbol, for example, where the first half is identical to the second one (in time order), except for a phase shift caused by the carrier frequency offset, then the normalized frequency offset estimate is

$$
\widehat{\phi}=\operatorname{angle}(P) .
$$

Subcarrier spacing for $802.11 \mathrm{n}$ is $312.5 \mathrm{KHz}$. Assuming a $25 \mathrm{ppm}$ local oscillator and a carrier frequency of $2.4 \mathrm{GHz}$, the signal can experience a CFO of less than \pm 0.6 times the subcarrier spacing. Thus, the integer estimation of the CFO can be avoided. Similar calculations and conclusions can be obtained for 802.16d.

LTE does not include a preamble in its frame, so a blind method should be used to accomplish CFO acquisition. Subcarrier spacing in LTE systems is $15 \mathrm{KHz}$; thus, normalized CFO can be higher than one. According to [12], first the fractional part of the CFO can be estimated by using the CP allocated in the OFDM symbol as shown in (4) and (5), where $r_{m}$ and $r_{m+L}$ are now the cyclic prefix and its copy, and $L=N$. After that, integer estimation can be performed in the frequency domain by using a modification of the algorithm described in [12]:

$$
\begin{gathered}
x_{k}=c p_{l, k} \cdot p_{l, k}, \\
\hat{n}_{I}=\arg \max \left|\sum_{\substack{k \in c p+m \\
m \in I}} x_{k}\right|,
\end{gathered}
$$

where $c p_{l, k}$ are the received pilot subcarriers inserted in the $l$ th OFDM symbol, $p_{l, k}$ are the known values of the pilot subcarriers, and $I$ is determined from $\left[-n_{\max }, n_{\max }\right]$. Due to the LTE pilot subcarrier structure, $n_{\max }=5$. By using the known values of the pilot subcarriers in (7), the integer part of the CFO can be calculated using only the first OFDM $\operatorname{symbol}(l=1)$.

The DVB-T/H frame does not include a preamble and it also has pilot subcarriers in the first OFDM symbol likewise LTE, so a similar approach to LTE acquisition can be used. The main difference between integer estimation in LTE and DVB-T/H is the length of the cyclic prefix and the number of pilot subcarriers that can vary depending on the transmission mode, thus increasing or decreasing the CFO estimation performance and its computational complexity.

It can be concluded that the same algorithm (4) and (5) can be applied in the four standards for fractional CFO acquisition by using the CP or the available preamble, whereas a similar method (6) and (7) can be used for integer acquisition in LTE and DVB-T/H where it is needed. Since algorithm reuse can be accomplished easily in the acquisition stage, the rest of the paper will focus on the tracking stage.

\section{CFO Tracking Schemes}

After acquisition, there still remains a little variation in the residual CFO. If that variation is not tracked and corrected, constellation points will fall in a different quadrant after a number of OFDM symbols, thus significantly degrading the system performance. For example, a residual $\mathrm{CFO}=0.02$ introduces a subcarrier rotation of $22^{\circ}$ after three OFDM 
symbols for a DVB $2 \mathrm{~K}$ mode with $\mathrm{CP}=64$ and $\mathrm{QPSK}$ constellation. Thus, accuracy and speed of convergence are important when implementing the CFO tracking closed loop. Although, this residual CFO also introduces ICI, it can be considered negligible in most cases, depending on the conditions and specifications. Therefore, the tracking effort should be aimed at correcting $\mathrm{CFO}$ rotation.

It should be mentioned that for DVB-T/H, channel estimation and equalization could be performed during all the data transmission by using the continual pilot subcarriers. This equalization would also correct partially the residual CFO rotation. However, even for this standard a residual CFO tracking scheme is highly recommended [12]. The CFO tracking scheme will be more critical for packetswitched systems, as $802.16 \mathrm{~d}$ and $802.11 \mathrm{n}$, where channel estimation is performed only at the beginning of the frame by using the preamble.

The so-called decision-directed methods (non-dataaided methods) compare the received data subcarriers with sliced versions (as fed from the demapper) to give a larger number of estimates. The Decision-Directed TimeFrequency Loop (DD-TFL) proposed in [15] for CFO tracking in the $802.11 \mathrm{~g}$ standard is based on two feedback loops in the time and the frequency domain and it uses all the data subcarriers to perform the estimations. Adaptations of this scheme for the 802.16d standard are found in [16] where the Decision-Directed Frequency Loop (DD-FL) and Data-Aided Frequency Loop (DA-FL) schemes are presented. DD-FL avoids the use of the time loop and uses less number of subcarriers per symbol to perform the tracking stage. By using DA-FL, the pilot subcarriers inserted in the data stream are used instead of the data subcarriers to perform the CFO estimations. DA-FL and DD-FL aim at reducing the CFO tracking computational complexity with almost no performance penalty. Other CFO tracking methods can be found in the literature as the classical scheme presented in [12]. However this DA tracking scheme requires pilot subcarriers in two consecutive OFDM symbols and this condition is not met by LTE. Therefore, this method is not considered in this work.

DA-FL, DD-FL, and DD-TFL can be adapted to other standard frames. The $802.11 \mathrm{n}, 802.16 \mathrm{~d}$, and DVB-T/H frames include pilot subcarriers in every OFDM symbol, whereas LTE includes pilot subcarriers in some specific symbols. Therefore, DA-FL performance is expected to worsen for this standard.

The DA-FL scheme [16] uses pilot subcarriers inserted in the OFDM data symbols. Its structure is represented in Figure 6.

The sequence $c_{k, l}$ after the FFT at the receiver is modified at every subcarrier as

$$
c_{k, l}^{\prime}=c_{k, l} e^{-j \Psi_{k, l}}, \quad 0 \leq k \leq N .
$$

The corrected data symbols $c_{k, l}^{\prime}$ may then be demapped to a bit stream. In the phase error detector (PED), the subcarrier pilots, $p_{k, l}$, are used for extracting the error increment $E_{k, l}$ according to one of the algorithms proposed in [18]. In particular, the algorithm selected here to extract the error increment computes

$$
\begin{gathered}
e_{k, l}^{I}=\operatorname{imag}\left(p_{k, l}\right)-\operatorname{imag}\left(p_{k, l}^{\prime}\right), \\
e_{k, l}^{Q I}=\operatorname{real}\left(p_{k, l}\right)-\operatorname{real}\left(p_{k, l}^{\prime}\right), \\
E_{k, l}=e_{k, l}^{Q} \operatorname{sgn}\left(\operatorname{real}\left(p_{k, l}\right)\right)-e_{k, l}^{I} \operatorname{sgn}\left(\operatorname{imag}\left(p_{k, l}\right)\right),
\end{gathered}
$$

where $p_{k, l}^{\prime}$ are the known values of the pilot subcarriers and $\operatorname{sgn}()$ is the sign function. After error extraction, the error increment $E_{k, l}$ is attenuated and enters the filter directly. Then, the estimated phase error $\Psi_{k, l}$ is applied to the post-FFT data symbol $c_{k, l}$. Therefore, CFO correction is updated as many times as pilot subcarriers are inserted in the OFDM symbol. Since this scheme performs correction in the frequency domain, it corrects the phase rotation and not the ICI introduced by the CFO. An important point to remark is that by using algorithm described in $(9,10,11)$ no complex multiplications are needed. This is an important improvement over classical tracking schemes as in [12].

The structure of the DD-FL scheme [16] is represented in Figure 7. This scheme also uses the error extraction algorithm described by (9). These equations are adapted to a decision-directed scheme by substituting the pilot subcarriers data $\left(p_{k, l}\right)$ by the data samples, and the known value of the pilot subcarriers $\left(p_{k, l}^{\prime}\right)$ by the samples at the output of the decisor.

The DD-TFL scheme [15] is composed of two tracking loops as it can be observed in Figure 8. The frequency loop uses the information provided by the output of the decisor to build the tracking system. In the time loop, the error $E_{k, l}$ estimated by the decision-directed phase error detector (DD-PED) is fed to the time branch and is averaged before entering the filter. As a result, the pre-FFT sample $r_{n, l}$ is rotated as

$$
r_{k, l}^{\prime}=r_{k, l} e^{-j(n+N g+l N s) \Psi_{l}}, \quad 0 \leq n \leq N .
$$

This time branch is able to correct the ICI introduced by the residual CFO; thus, a better performance is expected when compared to DD-FL.

These tracking schemes can be used on the four standards. The two DD methods can use all or some of the available data subcarriers to perform the tracking. In this work, all data subcarriers are used for $802.11 \mathrm{n}$, eight data subcarriers are used for $802.16 \mathrm{~d}$, every 6th subcarrier is used for LTE, and every 38th subcarrier is used for DVB-H/D. By choosing these values, simulations provide meaningful results and simulation times are not prohibitive. The DA method uses all the pilot subcarriers available in the frame.

\section{BER Results}

BER results for the complete synchronization system are obtained for each standard. A Rayleigh channel consisting of two paths is considered. The channel is perfectly estimated at the receiver and it is corrected using zero-forcing equalization. There is no coding of the QPSK signal, so performance 


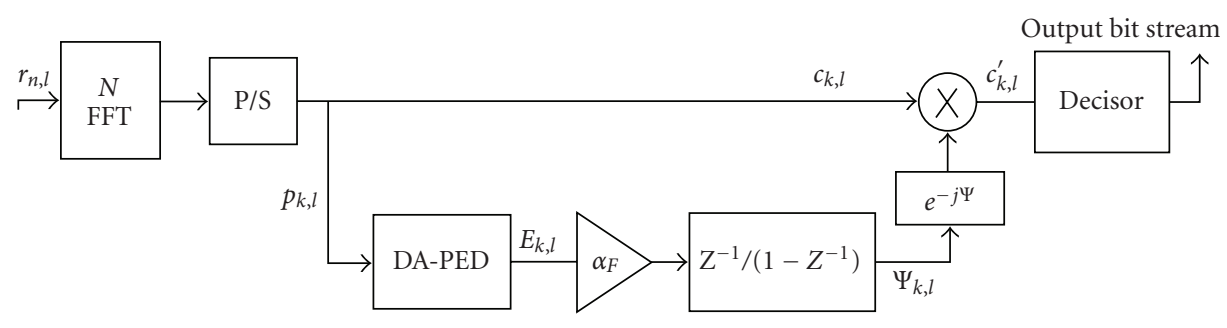

FIGURE 6: DA-FL scheme.

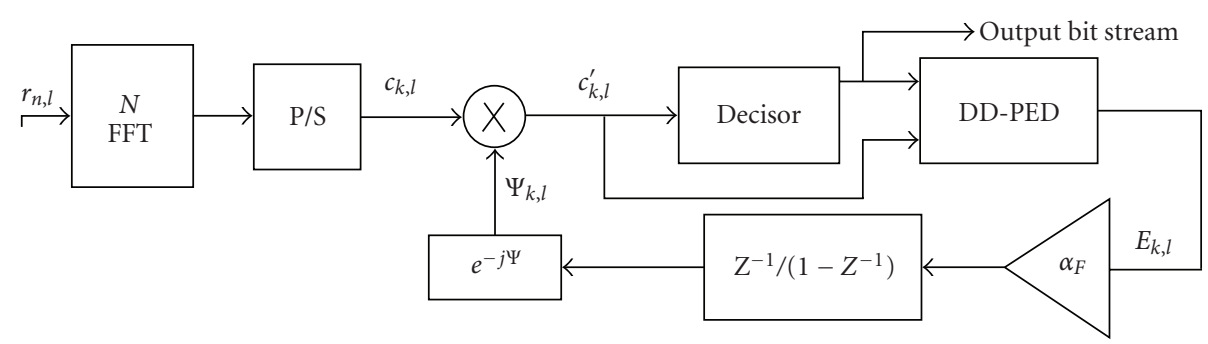

Figure 7: DD-FL scheme.

of the different schemes is shown through raw BER values. It is assumed that timing synchronization is perfectly achieved. The BER values are calculated by averaging the error bits throughout 10000 frames.

The $802.11 \mathrm{n}$ frame is simulated considering a system with a 64-point FFT and four pilots per symbol. The CP is composed of 16 samples. Each frame is composed of 100 OFDM symbols and the normalized CFO introduced in the system is 0.6. Similar length frame and normalized CFO are used for $802.16 \mathrm{~d}$. This standard requires a 256-point FFT and $N_{g}=32$ is used. In the case of LTE, the frame is composed of 140 OFDM symbols with an FFT size of 512, $N_{g}=64$ and $\mathrm{CFO}=2.7$. Finally, in the case of DVB-H, the frame is composed of 40 OFDM symbols with a 4048 point FFT, $N_{g}=128$, and a normalized frequency offset of 2.7. Table 1 summarizes the chosen parameters for the different standards. First of all, some previous simulations were performed to find the appropriate attenuation $\left(\alpha_{T}, \alpha_{F}\right)$ of the filters of the loops. Table 2 collects the values finally selected. Once the optimum attenuation values for the different schemes and standards were found, the BER results were obtained for a system where both CFO acquisition and tracking were enabled. Acquisition was performed for each standard as explained in Section 4, whereas three different tracking schemes (DA-FL, DD-FL, and DD-TFL) were evaluated for each standard.

Figure 9 shows the BER results for 802.11n. DA-FL obtains the best response and, for low noise values, DD-FL and DD-TFL approximate to the offset free case as well. This is because DD schemes rely on hits in the decisor block to work correctly. Hence, when noise decreases and less errors occur at the decisor, DD performance increases.

Figure 10 displays the results for $802.16 \mathrm{~d}$. The DA-FL scheme improves the BER obtained by the DD schemes. In a similar way to $802.11 \mathrm{n}$, the DD schemes approximate to DA-FL performance when the noise decreases. It is possible
TABLE 1: Parameters for the different standards.

\begin{tabular}{lcccc}
\hline & $802.11 \mathrm{n}$ & $802.16 \mathrm{~d}$ & LTE & DVB-T/H \\
\hline$N_{\text {FFT }}$ & 64 & 256 & 512 & 4048 \\
$N_{g}$ & 16 & 32 & 64 & 128 \\
Ts (us) & 4 & 72 & 83 & 448 \\
CFO & 0.6 & 0.6 & 2.7 & 2.7 \\
$\begin{array}{l}\text { Pilot subcarriers } \\
\text { per OFDM }\end{array}$ & 4 & 8 & 50 & 89 \\
$\begin{array}{l}\text { symbol } \\
\begin{array}{l}\text { Data subcarriers } \\
\text { in DD schemes }\end{array}\end{array}$ & 48 & 8 & 50 & 89 \\
$\begin{array}{l}\text { Frame length } \\
\text { (OFDM }\end{array}$ & 100 & 100 & 140 & 40 \\
symbols) & & & & \\
\hline
\end{tabular}

TABLE 2: Optimal loop parameters.

\begin{tabular}{|c|c|c|c|}
\hline & DA-FL & DD-FL & DD-TFL \\
\hline $802.11 \mathrm{n}$ & $\alpha_{F}=7 \times 10^{-2}$ & $\alpha_{T}=5 \times 10^{-5}$ & $\alpha_{F}=5 \times 10^{-5} \quad \alpha_{T}=10^{-3}$ \\
\hline $802.16 \mathrm{~d}$ & $\alpha_{F}=2 \times 10^{-4}$ & $\alpha_{T}=10^{-4}$ & $\alpha_{F}=10^{-4} \quad \alpha_{T}=10^{-5}$ \\
\hline LTE & $\alpha_{F}=10^{-4}$ & $\alpha_{T}=10^{-5}$ & $\alpha_{F}=10^{-5} \quad \alpha_{T}=10^{-3}$ \\
\hline DVB-T/H & $\alpha_{F}=10^{-2}$ & $\alpha_{T}=10^{-2}$ & $\alpha_{F}=10^{-2} \quad \alpha_{T}=10^{-3}$ \\
\hline
\end{tabular}

to improve DD performance in this case by increasing the number of data subcarriers used in the tracking estimation. However, this would also increase the computational requirements.

Figure 11 shows the plot for LTE. It can be observed that DA-FL performance is unacceptable, while DD schemes obtain BER values close to the offset free case. This is because there are no pilots inserted in every OFDM symbol, so tracking convergence is not fast enough for DA-FL. Thus, this standard encourages the use of DD methods. As it was 


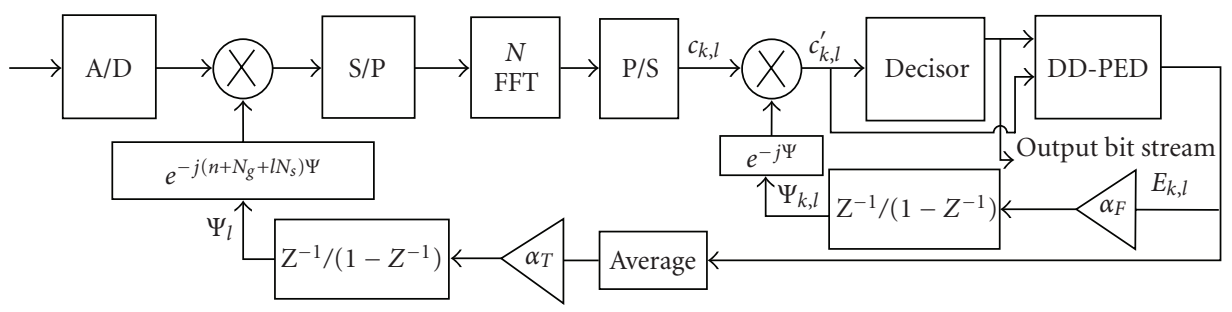

Figure 8: DD-TFL scheme.

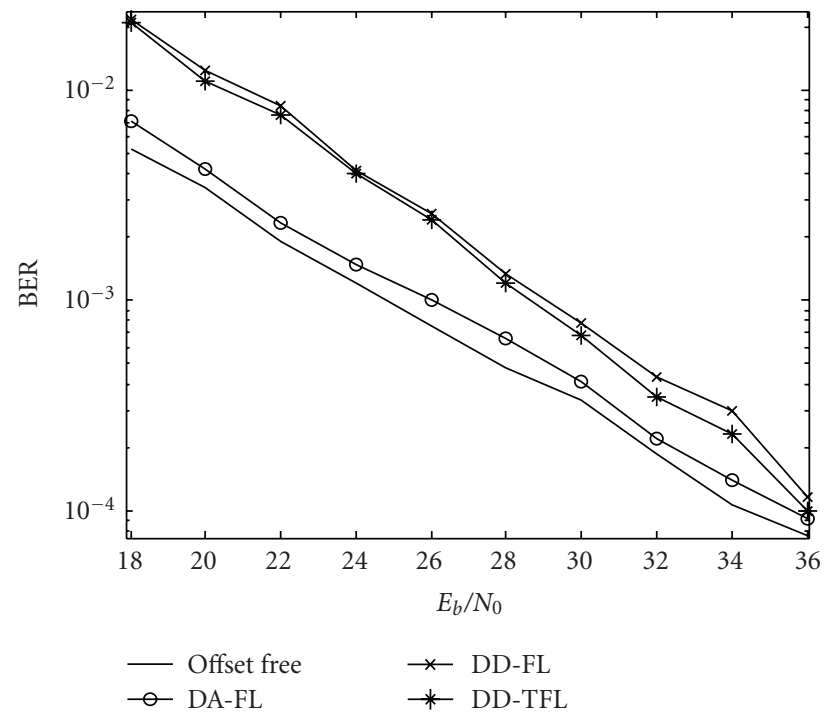

Figure 9: BER values for 802.11n.

expected, DD-TFL behaves better than DD-FL although the difference is small.

Figure 12 displays the results for DVB-T/H. The DA-FL scheme clearly outperforms the DD schemes. That is due to the "small" number of data subcarriers used for CFO tracking. It is possible to improve the DD performance, similarly to $802.16 \mathrm{~d}$ and LTE by increasing the number of data subcarriers and the computational complexity.

Therefore, from the previous performance results it can be concluded that DD-TFL is the best option for a common implementation for the three standards since it improves slightly the DD-FL performance and DA-TL has an unacceptable performance for LTE.

\section{Implementation Issues}

The BER performance of the different schemes has been shown in Section 6. However, there still remains an important issue that needs to be considered for implementation purposes: their computational complexity. This is a key issue when determining the number of hardware resources needed for portable, battery-powered systems. Computations are described in terms of real multiplications (M), additions $(S)$, and multiplications by a constant (MC). A complex multiplication is implemented using $3 \mathrm{M}$ and $5 \mathrm{~S}$. CFO correction is implemented through a complex multiplication. On the

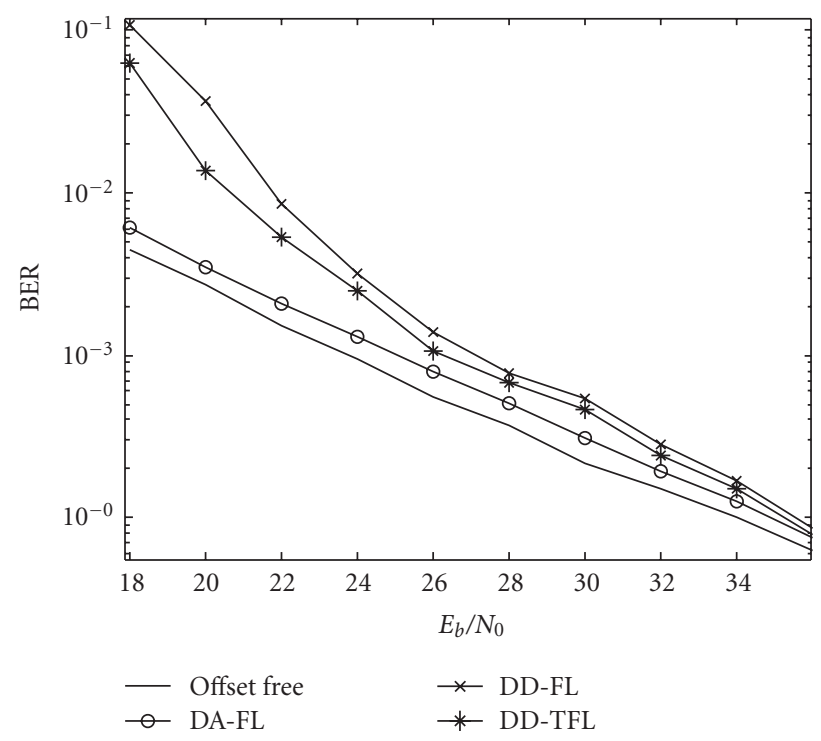

FIGURE 10: BER values for $802.16 \mathrm{~d}$.

TABLE 3: Number of operations and resources.

\begin{tabular}{ccccc}
\hline & & DA-FL & DD-FL & DD-TFL \\
\hline \multirow{4}{*}{ Ops } & $802.11 \mathrm{n}$ & $36 / 75 / 14$ & $36 / 108 / 36$ & $72 / 180 / 12$ \\
& $802.16 \mathrm{~d}$ & $9 / 19 / 2$ & $9 / 21 / 4$ & $18 / 40 / 4$ \\
& LTE & $11 / 20 / 2$ & $11 / 23 / 4$ & $21 / 42 / 4$ \\
& DVB-T/H & $22 / 37 / 1$ & $22 / 37 / 1$ & $44 / 74 / 1$ \\
\hline \multirow{4}{*}{ Res } & $802.11 \mathrm{n}$ & $6 / 9$ & $6 / 9$ & $12 / 17$ \\
& $802.16 \mathrm{~d}$ & $6 / 9$ & $6 / 9$ & $12 / 17$ \\
& LTE & $6 / 9$ & $6 / 9$ & $12 / 17$ \\
& DVB-T/H & $6 / 9$ & $6 / 9$ & $12 / 17$ \\
\hline
\end{tabular}

other hand, the required FPGA resources are described in terms of embedded multipliers and adders (EM/A).

Table 3 describes the three synchronization schemes for each standard according to their (M/S/MC) computations, as millions of operations per second, and their required (EM/A) resources. The computations per second are calculated taking into account the operations performed by each method, including the algorithm, the filter, and the correction, and considering the bit rates defined in the standards. The required resources are obtained by scheduling the operations involved assuming that they are performed iteratively subcarrier by subcarrier. No other sharing of resources has been considered in the architecture. 


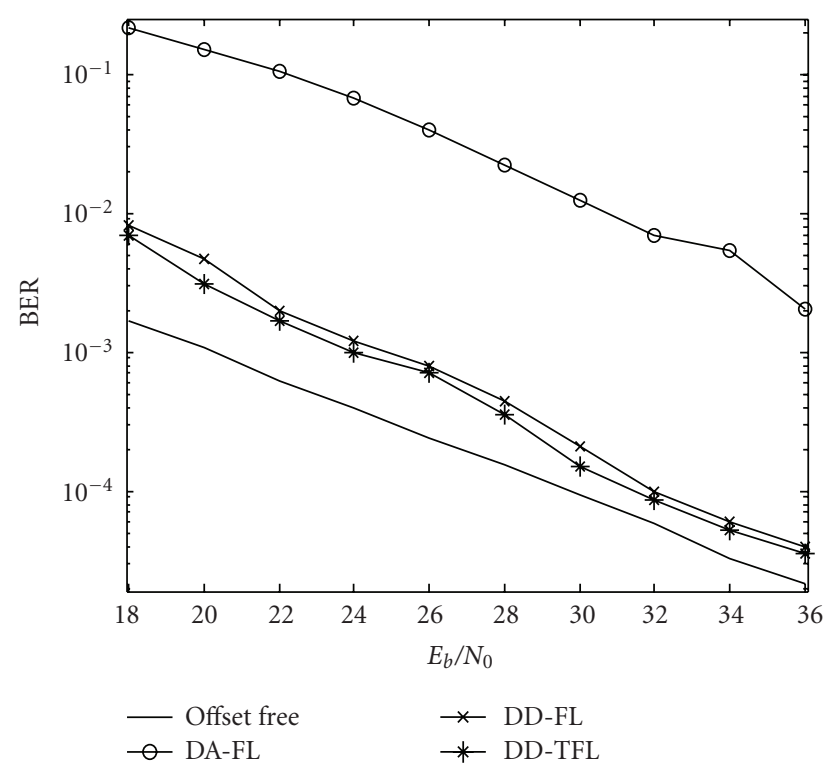

FIgURE 11: BER values for LTE.

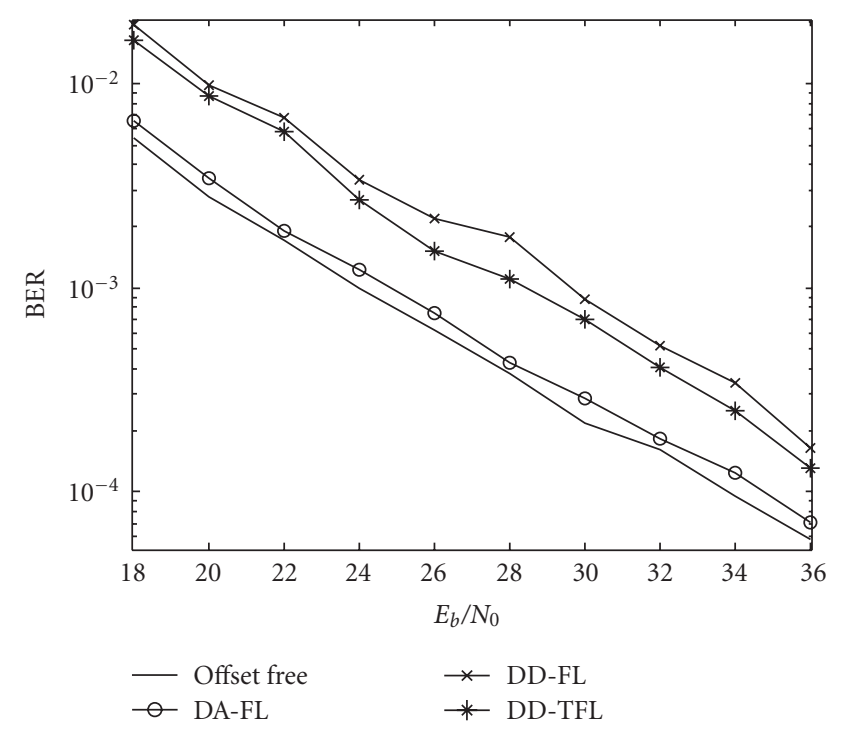

Figure 12: BER values for DVB-T/H.

It can be observed that DA-FL and DD-FL need less than a half of the number of operations required by DDTFL. Therefore, DD-TFL not only would require more resources, but also would consume more power. In this framework, a new analysis of the results obtained in Section 6 reveals that the advantage of DD-TFL over DD-FL can be considered negligible. It is also important to note that DDFL and DD-TFL will increase or reduce their computations (and also their performance) depending on the actual number of data subcarriers in the OFDM symbol. Therefore, when considering computational requirements in addition to performance, it turns out that the best alternative is $\mathrm{DD}$ FL.

Nevertheless, an even better solution can be found by looking at the structure of the three tracking schemes. Since
TABLE 4: Features of the three solutions.

\begin{tabular}{lccc}
\hline & DD-TFL & DD-FL & DA-FL \& DD-FL \\
\hline EM (\% total) & $18 \%$ & $9 \%$ & $9 \%$ \\
RE (\% time) & $84 \%$ & $2 \%$ to $59 \%$ & $2-3 \%$ \\
dB losses & 1.6 to 3 & 1.8 to 3.6 & 0.5 to 1.8 \\
\hline
\end{tabular}

DA-FL and DD-FL use the same estimation algorithm, both schemes can be implemented using the same resources and work for the four different frames (DA-FL for 802.11n, 802.16d, and DVB-T/H, and DD-FL for LTE). To accomplish that, only two memories with the number and position of the pilot or data subcarriers involved in the tracking are needed to switch between DA-FL and DD-FL. This solution also offers more possibilities to reuse the EMs available in the FPGA.

Table 4 summarizes the three possible multistandard solutions considering that the target device is a Virtex 4 xc4vlx60 which contains $66 \mathrm{EM}$. For each solution, it includes the percentage of EMs used in the FPGA, the resource utilization (RE) described as a percentage of the total time, and the range of signal losses in $\mathrm{dB}$ for a target $\mathrm{BER}=$ $10^{-4}$. In the case of resource utilization, the percentages are obtained from the ratio of the subcarriers that are being used to calculate the CFO estimates with respect to the total number of subcarriers available in each OFDM symbol. These percentages somehow describe the possibilities of further resource reuse. Some values in the table are given as ranges that include the results for the four standards being evaluated. For example, the DD-FL solution allows a $2 \%$ resource utilization for DVB-T/H, 3\% for $802.16 \mathrm{~d}, 10 \%$ for LTE, and $59 \%$ for $802.11 \mathrm{n}$.

\section{Conclusions}

In this work, a comparison of different frequency synchronization schemes for four wireless communications standards (802.11n, 802.16d, LTE, and DVB-T/H) has been presented, aimed at a multistandard FPGA implementation. Focus is on the tracking stage, as acquisition is performed using the same algorithm for $802.11 \mathrm{n}, 802.16 \mathrm{~d}$, LTE, and DVB-T/H. In the case of $802.11 \mathrm{n}$ and $802.16 \mathrm{~d}$, only fractional CFO acquisition is performed over the preamble.

Despite the frame differences between the standards, three different methods to accomplish CFO tracking have been evaluated. DA-FL performs well for $802.11 \mathrm{n}, 802.16 \mathrm{~d}$, and DVB-H/T. However, DA-FL performance for LTE is unacceptable due to the fact that no pilot subcarriers are inserted at each OFDM symbol. DD-TFL is the scheme with best performance for the four standards but, after analyzing the computational requirements and the possibilities of resource reuse, DD-FL appears as a more balanced solution. Furthermore, a solution that combines DA-FL for $802.11 \mathrm{n}, 802.16 \mathrm{~d}$ and DVB-T/H standards and DD-FL for LTE by including a small additional memory to switch between standards has been proposed, showing overall better performance than DD-TFL and requiring only half of its resources. 


\section{Acknowledgment}

The work presented in this paper has been supported in part by the Spanish Ministry of Science and Innovation under projects no. TEC2006-13067-C03-03 and no. TEC200914219-C03-02 and by the European Commission under the FP7-ICT project MULTI-BASE (216541).

\section{References}

[1] "IEEE draft standard for information technology-telecommunications and information exchange between systems-local and metropolitan area networks-specific equirements-part 11: wireless lAN medium access control (MAC) and physical layer (PHY) specifications amendment: enhancements for higher throughput," June 2009.

[2] "IEEE standard for local and metropolitan area networks part 16: air interface for fixed broadband wireless access systems," IEEE 802.16, 2004.

[3] A. B. Ericsson, "Long term evolution (LTE): an introduction," White paper, October 2007.

[4] ETSI EN 300 744, "Digital video broadcasting (DVB): frame structure, channel coding and modulation for digital terrestrial television (DVB-T)," Tech. Rep., ETSI, 2004.

[5] DVB-H-Transmission Systems for Handheld Terminals- EN 302204 v1.1.1, http://www.dvb-h.org/.

[6] C. Garuda and M. Ismail, "A multi-standard OFDM-MIMO transceiver for WLAN applications," in Proceedings of the 48th IEEE International Midwest Symposium on Circuits and Systems, pp. 1613-1616, Cincinnati, Ohio, USA, August 2005.

[7] B. Mennenga, J. Guo, and G. Fettweis, "A component based reconfigurable baseband architecture," in Proceedings of the 16th IST Mobile and Wireless Communication Summit, pp. 1-5, Budapest, Hungary, July 2007.

[8] R. Barrak, A. Ghazel, and F. Ghannouchi, "Optimized multistandard rf subsampling receiver architecture," IEEE Transactions on Wireless Communications, vol. 8, no. 6, pp. 2901-2909, 2009.

[9] F. Gallazi, G. Torlli, P. Malcovati, and V. Ferragina, "A digital multistandard reconfigurable FIR filter for wireless applications," in Proceedings of the 14th IEEE International Conference on Electronics, Circuits and Systems, pp. 808-811, Marrakech, Morocco, December 2007.

[10] J.-J. van de Beek, M. Sandell, and P. O. Borjesson, "ML estimation of time and frequency offset in OFDM systems," IEEE Transactions on Signal Processing, vol. 45, no. 7, pp. 18001805, 1997.

[11] T. Schmidl and D. Cox, "Robust frequency and timing synchronization for OFDM," IEEE Transactions on Communications, vol. 45, no. 12, pp. 1613-1621, 1997.

[12] M. Speth, S. Fechtel, G. Fock, and H. Meyr, "Optimum receiver design for OFDM-based broadband transmissionpart II: a case study," IEEE Transactions on Communications, vol. 49, no. 4, pp. 571-578, 2001.

[13] P. Moose, "A technique for orthogonal frequency division multiplexing frequency offset correction," IEEE Transactions on Communication, vol. 42, pp. 2901-2914, 1994.

[14] G. Santella, "A frequency and symbol synchronization system for OFDM signals: architecture and simulation results," IEEE Transactions on Vehicular Technology, vol. 49, no. 1, pp. 254275,2000 .
[15] L. Kuang, Z. Ni, J. Lu, and J. Zheng, "A time-frequency decision-feedback loop for carrier frequency offset tracking in OFDM systems," IEEE Transactions on Wireless Communications, vol. 4, no. 2, pp. 367-373, 2005.

[16] J. González-Bayón, C. Carreras, and A. Fernández-Herrero, "Comparative evaluation of carrier frequency offset tracking schemes for WiMAX OFDM systems," in Proceedings of the IEEE Symposium on Signal Processing and Information Technology (ISSPIT '07), Cairo, Egypt, December 2007.

[17] M. Speth, S. Fechtel, G. Fock, and H. Meyr, "Optimum receiver design for wireless broadband systems using OFDM-part I," IEEE Transactions on Communictions, vol. 47, pp. 1668-1677, 1999.

[18] S. Moridi and H. Sri, "Analysis of four decision-feedback carrier recovery loops in the presence of intersymbol interference," IEEE Transactions on Communictions, vol. 33, pp. 543$550,1985$. 

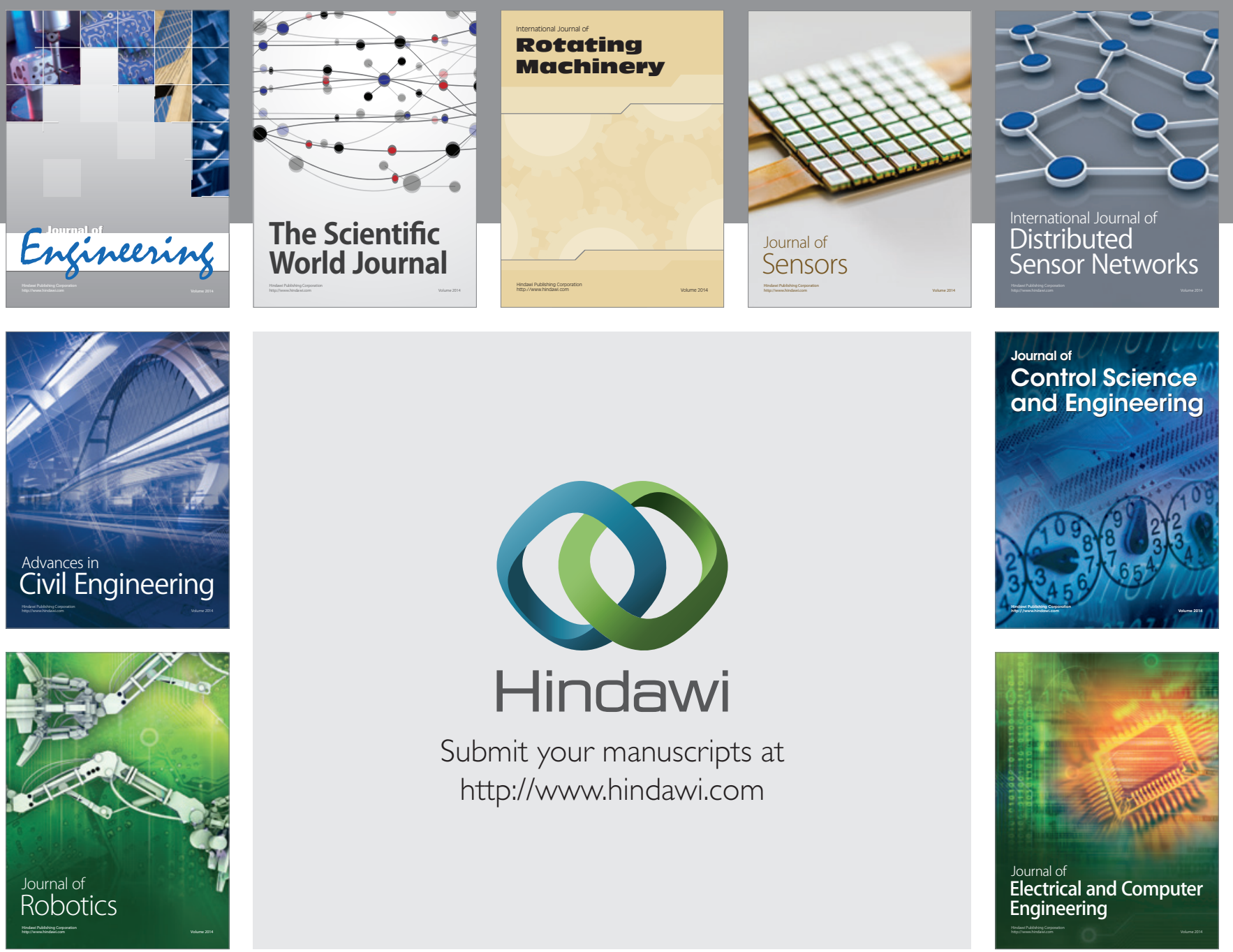

Submit your manuscripts at

http://www.hindawi.com
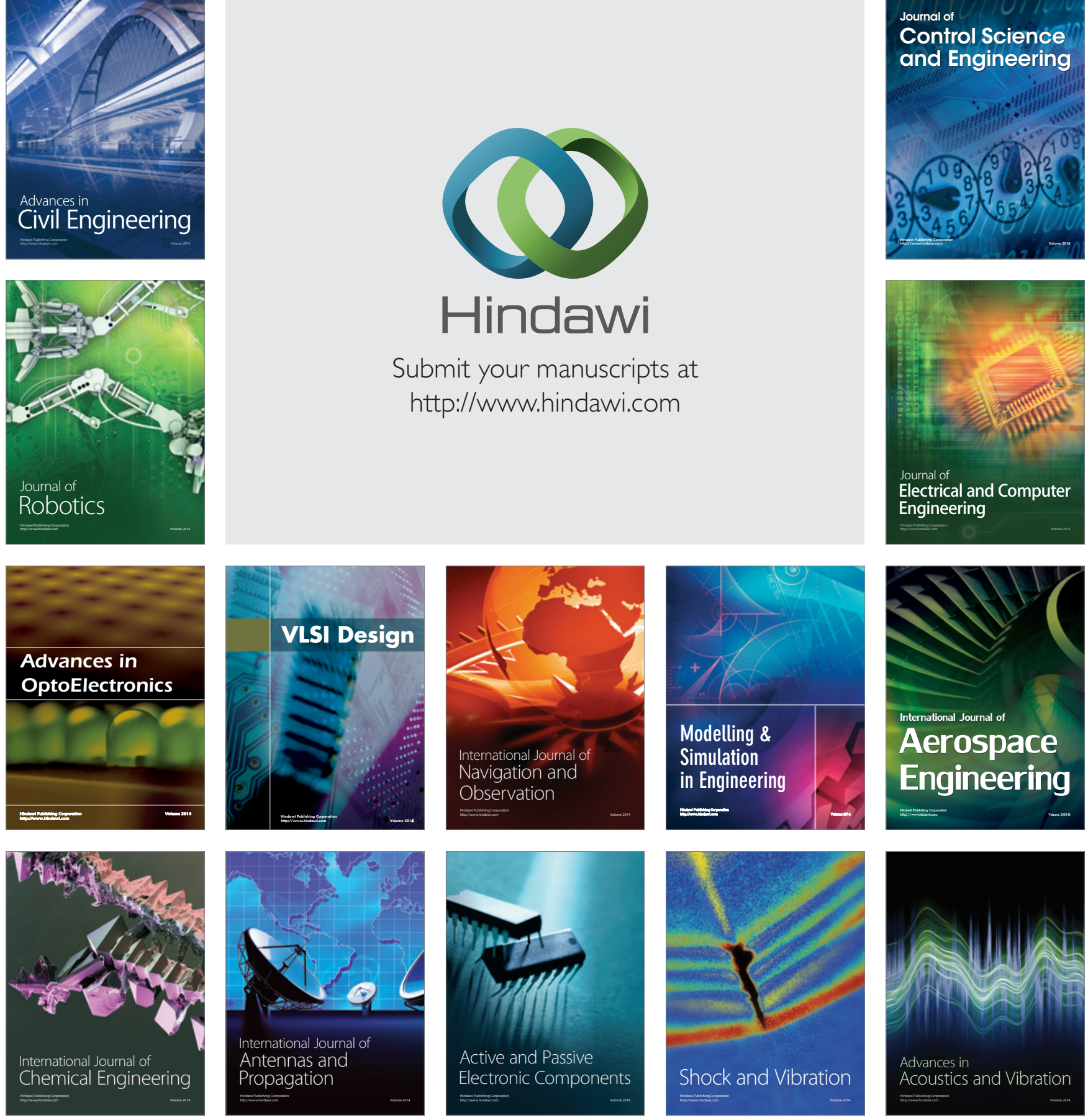